Review Article

\title{
Acupuncture for Primary Dysmenorrhea: A Potential Mechanism from an Anti-Inflammatory Perspective
}

\author{
Wen-Yan Yu, ${ }^{1}$ Liang-Xiao Ma $\mathbb{D}^{1,2}$, Zhou Zhang, ${ }^{1}$ Jie-Dan Mu, ${ }^{1}$ Tian-Yi Sun, ${ }^{1}$ Yuan Tian, ${ }^{1}$ \\ Xu Qian, ${ }^{1}$ and Yi-Dan Zhang ${ }^{1}$ \\ ${ }^{1}$ School of Acupuncture, Moxibustion and Tuina, Beijing University of Chinese Medicine, Beijing 100029, China \\ ${ }^{2}$ The Key Unit of State Administration of Traditional Chinese Medicine, Evaluation of Characteristic Acupuncture Therapy, \\ Beijing 100029, China
}

Correspondence should be addressed to Liang-Xiao Ma; maliangxiao@vip.sina.com

Received 6 August 2021; Revised 7 September 2021; Accepted 26 November 2021; Published 3 December 2021

Academic Editor: Liming Lu

Copyright (C) 2021 Wen-Yan Yu et al. This is an open access article distributed under the Creative Commons Attribution License, which permits unrestricted use, distribution, and reproduction in any medium, provided the original work is properly cited.

The low adverse effects of acupuncture for primary dysmenorrhea (PD), known as one of the most commonly reported gynecological debilitating conditions affecting women's overall health, have been thus far confirmed. Moreover, it has been increasingly recognized that inflammation is involved in such menstrual cramps, and recent studies have further shown that the anti-inflammatory effects of acupuncture are helpful in its control. This review portrays the role of inflammation in PD pathophysiology, provides evidence from clinical and animal studies on acupuncture for inflammation-induced visceral pain, and reflects on acupuncture-related therapies for dysmenorrhea with regard to their anti-inflammatory characteristics. Further research accordingly needs to be carried out to clarify the effects of acupuncture on proinflammatory factors in PD, particularly chemokines and leukocytes. Future studies on this condition from an anti-inflammatory perspective should be also performed in line with the notion of emphasizing stimulation modes to optimize the clinical modalities of acupuncture. Additionally, the effects and mechanism of more convenient self-healing approaches such as TENS/TEAS for PD should be investigated.

\section{Introduction}

Primary dysmenorrhea (PD), also called functional painful periods or menstrual cramps, is manifested as lower abdominal pains during menstruation without pelvic pathologies, accompanied by symptoms such as lumbago and leg pain, diarrhea, nervousness, fatigue, loss of appetite, and nausea and vomiting. PD usually occurs in adolescence after the menarche, and the pain starts a few hours before or immediately after menstruation and typically lasts for 8-72 hours $[1,2]$. According to the latest epidemiological survey, about $45-90 \%$ of women in the world suffer from this condition during their menstrual period, among which $10-25 \%$ are of severe types [3]. Therefore, PD seems to be one of the most common gynecological disorders regardless of nationality and age [4]. About $1 / 3-1 / 2$ of women with this debilitating disorder are also absent from work or school at least once per cycle, which significantly affects the quality of their work, study, and daily living activities, and even brings huge economic losses to any society $[5,6]$. In addition, the occurrence of PD augments the risk of chronic pelvic inflammatory disease [4]. Although nonsteroidal anti-inflammatory drugs (NSAIDs) are currently the first-line medication for PD with well-grounded efficacy, the ineffective rate can reach about $20-30 \%$ [7]. Therefore, there is an urgent clinical demand to find a low-risk and effective nonpharmacological treatment option to relieve menstrual cramps.

Acupuncture has been popularly applied for a variety of diseases in China and many other countries as well, particularly for pain management $[8,9]$. In fact, under the term "acupuncture", there is a family of treatment modalities, including manual acupuncture, electroacupuncture (EA), moxibustion, acupoint catgut embedding (ACE) treatment, transcutaneous electrical acupoint stimulation (TEAS), transcutaneous electrical nerve stimulation (TENS) [10]. A 
large number of clinical trials and reviews have further provided evidence in support of acupuncture and its related therapies for PD [2, 11-15].

This alternative medicine has also demonstrated promising anti-inflammatory effects; therefore, it has been applied to treat inflammatory diseases [16-18], especially inflammatory pain [19-22]. Numerous studies have so far elucidated the underlying mechanism of acupuncture for various types of pain, including visceral pain [22, 23], and it has been emphasized that anti-inflammatory effects can contribute to the analgesic mechanism of this alternative therapy.

As a commonly seen among gynecological disorders, PD refers to a type of visceral pain, which develops closely related to inflammatory and immune factors [24-26]. An increasing number of studies have further examined the mechanism of acupuncture for PD from an anti-inflammatory perspective. This article aims to review the role of inflammation in PD pathophysiology and explore the potential anti-inflammatory analgesic mechanism of acupuncture as a form of alternative medicine to promote further research and optimize its clinical modalities.

\section{Role of Inflammation in PD Pathophysiology}

The menstrual cycle is manifested as a cyclical pattern of hormonal changes modulated by a feedback mechanism on the hypothalamus-pituitary-ovary (HPO) axis [27]. In the late secretory phase of the menstrual cycle, atrophic luteum accompanied by a rapid decline in hormone levels is the main regulatory factor shaping the destruction of the menstrual cascade [28]. Progesterone withdrawal also leads to the release of acid phosphatase and lysozyme from lysosomes into the cytoplasm. Arachidonic acid (AA) is further metabolized to prostaglandins (PGs) and leukotrienes (LTs) via the cyclooxygenase (COX) and lipoxygenase (LOX) pathways, respectively, both contributing to excessive myometrial contractions, giving rise to ischemia and hypoxia in the uterine muscle tissues [29]. The etiology of PD is multifaceted. Although numerous studies have been so far done in this respect, the pathophysiology of this alternative therapy has not been still fully clarified. The most generally acknowledged explanation is the concentration of PGs during menstruation [30]. In addition, a range of events including age, smoking habits, age at menarche, body mass index (BMI), alcohol abuse, family history, exercise, and the like can be among the risk factors for PD [4].

Notably, it has been suggested that menstruation is an inflammatory process $[24,25]$, before it, the endometrium exhibits inflammatory features of red with blood and edematous tissues, as a phenomenon associated with an influx of proinflammatory cytokines (viz. Interleukin-1 [IL1], IL-6, and tumor necrosis factor-alpha $[\mathrm{TNF} \alpha]$ ) and leukocytes [28]. The inflammatory mediators are also an important part of the menstrual process [26], driven by a decrease in the levels of nonsteroid hormones in the late secretory phase of a nonconceptual cycle [28]. Based on previous studies, the relationship between inflammation and PD has recently attracted increasing attention even though people have a deeper understanding of the occurrence and development of PD. In the following, the relationship between PD pathophysiology and inflammation is being discussed.

\subsection{Progesterone Withdrawal-Initiated Inflammation in} Menstrual Cycle in PD. The current inflammatory perspective of the menstrual cycle in PD involves a complex set of events, driven by the fall in progesterone levels, which activate the release of nuclear factor- $\kappa \mathrm{B}(\mathrm{NF}-\kappa \mathrm{B})$ from its inhibition by the inhibitor of $\mathrm{I} \kappa \mathrm{B}$, leading to the downstream transduction and translation of inflammatory genes and a release of proinflammatory mediators (that is, inflammatory cytokines, chemokines, and PGs) together with an influx of inflammatory cells [31-33]. Beyond that, a cascade of inflammatory events reflected in the activation of matrix metalloproteinases (MMPs) and other degradation enzymes can participate in maintaining inflammation and eventually cause tissue destruction.

Of note, progesterone plays an anti-inflammatory role in the menstrual cycle $[34,35]$. The continued presence of progesterone can significantly inhibit excessive inflammatory events in the endometrium [36-38]. Distinct uterine inflammation manifested by substantial leukocyte content has been also shown in mice lacking progesterone receptors [36]. Progesterone additionally inhibits the production and activation of MMPs [39] and reduces the inflammatory responses induced by T-cell activation [35]. The reduction of ovarian hormone levels in the endometrium is typically associated with PD [40]. One other study have further revealed that imbalances in estradiol and progesterone could also affect the synthesis of PGF2 $\alpha$ in the endometrium and lead to menstrual pain [41]. Therefore, progesterone may enhance $\mathrm{PD}$ prognosis by regulating the levels of cytokines, inhibiting the activation and migration of immune cells, and reducing the oxidant activity. The inflammatory response caused by progesterone withdrawal in the first phase of the menstrual cycle is thus involved in PD occurrence.

\subsection{PGs-Mediated Inflammatory Response in PD. PGs are} associated with inflammatory effects and they are considered as the fundamental mechanism for PD formation [30]. PGF $2 \alpha$ can further cause vasoconstriction and result in a decrease in the blood flow, which in turn stimulate abnormal spastic contraction of uterine smooth muscles, and ultimately induce tissue ischemia and hypoxia, and pain [42]. The function of $\mathrm{PGE}_{2}$ depends on the type of receptors [43]. $\mathrm{PGE}_{2}$ mediated by the $\mathrm{PGE}_{2}$ receptor 2 also plays the role of relaxing vessels and inhibiting the contraction of uterine smooth muscles and may even work to increase edema and recruit leukocytes [44]. Studies in this line have reported that patients with PD experience significantly higher levels of PGF $2 \alpha$ and PGE2 in the endometrial and menstrual blood than those without this condition [30]. There is even evidence that PGF $2 \alpha$ can augment the sensitivity of nerve endings to pain and lower the pain perception threshold [45]. 
In addition, some studies have demonstrated that PGs can enhance the migration of inflammatory factors to the endometrium $[44,46] . \mathrm{PGF}_{2 \alpha}$ can further promote neutrophils (NEUT) migration by increasing the release of CXCL1 [47], while $\mathrm{PGE}_{2}$ enhances leukocyte migration by inducing the expression of CXCL8 via NF- $\kappa \mathrm{B}$ signaling pathway $[48,49]$. Additionally, the overexpression of inflammatory factors (such as TNF $\alpha$, IL-1, and IL-6) can elevate the synthesis or release of $\mathrm{PGF}_{2 \alpha}$ in the uterus to trigger PD [50].

2.3. LTs and Inflammation in PD. LTs, as important inflammatory mediators, play an essential role in the PD process [51, 52]. Studies have accordingly shown that $10-30 \%$ of patients with PD have no obvious changes in PG levels in the uterus, while the content of LTs in the uterus and menstrual blood had significantly increased, and the LTE4 content in the urine of some young PD patients on the first day of menstruation was equivalent to three times that of women without this condition $[53,54]$. LTs can also participate in the chemotaxis and activation of leukocytes, causing leukocytes to accumulate in the inflammatory area and release inflammatory mediators, which can lead to smooth muscle contractions and increased vascular permeability. Studies have further revealed that the use of LT antagonists can effectively relieve pain in patients with PD, whose traditional treatment with PG synthase inhibitors might not be much effective [55].

2.4. Changes of Cytokines and Chemokines in PD. The overexpression of cytokines and chemokines during the menstrual cycle drives the inflammatory microenvironment of PD in the uterus and plays a leading role in leukocyte recruitment.

2.4.1. TNF $\alpha$. TNF $\alpha$ is a potent proinflammatory cytokine that mediates complex biological responses, including the upregulation of inflammatory ones [50]. The role of TNF $\alpha$ in $\mathrm{PD}$ pathogenesis has been emphasized as stimulating the synthesis or release of PGs [50,56], resulting in hypercontraction of the myometrium, which leads to ischemic pain. Studies have further reported that women with PD have higher plasma IL- 6 and TNF $\alpha$ levels than healthy cases $[57,58]$. Targeting $\mathrm{TNF} \alpha$ and other factors to regulate arachidonic acid and inflammatory signaling pathways is thus assumed as an effective approach for PD treatment [59]. Moreover, the genotype of TNF $\alpha-308$ GG may be a useful tool for predicting PD susceptibility [60].

2.4.2. IL. Plasma cytokine levels, including IL- $1 \beta$, IL-6, and IL-10, have been significantly altered in women with a normal menstrual cycle [61]. IL-1 $\beta$ and IL-6 levels have been also negatively relevant to estradiol and progesterone levels, indicating the involvement of immune inflammation in the menstrual cycle [62]. Studies have additionally shown that the IL-6 level in the luteal phase was significantly higher than that in follicular one [63], and plasma IL-6 concentration significantly increased in patients with PD on the first day of menstruation, resulting in enhanced uterine muscle contractions and reduced uterine blood flow [64, 65]. Research has further found that a reduction in IL-6 levels and an increase in anti-inflammatory factors can be induced by aerobic exercises, therefore relieving PD [50].

2.4.3. Eotaxin. Eotaxin, also known as $\mathrm{C}-\mathrm{C}$ motif chemokine ligand 11 (CCL11), is a member of the CC subfamily of chemokines and acts after binding to $\mathrm{C}-\mathrm{C}$ motif chemokine receptor 3 (CCR3). As a specific chemokine of eosinophil (EOS), eotaxin has the strongest chemotactic activity [66] and it has been confirmed that the local injection of CCL11 can significantly augment EOS in local tissues [67]. A recent study has shown that patients with PD have significantly higher eotaxin levels in their blood than healthy individuals, suggesting that eotaxin may be involved in PD development [68].

During the menstrual cycle, cytokines and chemokines are abundant in the endometrium, which recruits leukocytes and affects their division and activation. By regulating the composition and function of local uterine leukocytes, cytokines and chemokines can further enhance and maintain local uterine inflammation, leading to tissue damage and indirect involvement in PD.

2.5. Changes of Leukocytes in PD. Menstruation represents a highly regulated inflammatory process, manifested as substantial leukocytes before the occurrence of menstruation [44]. The major leukocyte subsets, like uterine NEUT, natural killer (NK) cells, mast cells (MCs), EOS, and macrophages, constitute up to $40 \%$ of the total cells in the premenstrual endometrium [69]. There is also growing evidence that leukocytes are closely associated with PD.

2.5.1. NEUT. In the peripheral blood of patients with PD, inflammatory metabolites of NEUT are increased [58, 70]. Serum neutrophil-to-lymphocyte ratio is significantly higher in adolescents with PD and premenstrual syndrome (PMS) [71]. In this sense, NSAIDs can bring pain-relief effects via modulating oxidative stress and ionized calcium $\left(\mathrm{Ca}^{2+}\right)$ levels of NEUT in patients with PD through voltage-gated calcium channels (VGCCs) and transient receptor potential (TRP) cation ones [72].

2.5.2. EOS. In addition to the classic inflammatory response, allergic inflammation dominated by EOS is also of utmost importance and usually not recognized in PD pathophysiology. Recently, the role of EOS in the reproductive system has attracted much attention. Studies have further found that EOS is present in the endometrium before and during menstruation [73, 74]. Besides, EOS can regulate local immune and inflammatory responses and play a key role in PD induction and development. The elevated levels of eotaxin can also cause EOS migration to the uterus, promoting inflammatory edema and congestion in this organ [75]. Eosinophil cationic protein (ECP), major basic protein 
(MBP), neurotoxin, and eosinophil peroxidase released by activated EOS can also cause the release of reactive oxygen species (ROS) and cytotoxic molecules, promoting inflammation and inducing endothelial damage to activated platelets, which lead to vasoconstriction and blood clotting and ultimately reduced blood flow and aggravated PD. ECP can further promote MC release histamine and exacerbate pain [76]. Further explorations into the relationship between EOS and menstruation can thus help better understand the pathogenesis of PD.

2.5.3. MCs. Human MCs are derived from $\mathrm{CD}^{+} 4^{+}$and $\mathrm{CD} 117^{+}$pluripotent hematopoietic stem cells in the bone marrow. MC progenitor cells can be also transformed from protective immune cells to effective proinflammatory ones, thus participating in the inflammatory process of different tissues. They are additionally involved in the induction of acute inflammation and tissue repair during chronic inflammation. In the female reproductive system, MCs are mainly distributed in the myometrium and endometrium layers [77], which are closely related to uterine smooth muscles, fibroblasts, and collagens. The mediators released by MCs can effectively stimulate uterine smooth muscle contractions. Studies have accordingly shown that MC activation plays a critical role in the control of full-term and premature delivery $[78,79]$ and is essential in the progression of inflammatory bowel disease (IBD) [80], rheumatoid arthritis (RA) [81], and cutaneous vasculitis [82].

Therefore, inflammatory factors are directly linked with PD occurrence and development. In addition to classic paincausing substances (such as PGs and LTs), the inflammatory response contributes to $\mathrm{PD}$, mainly caused by cytokines (i.e., IL-6 and TNF $\alpha$ ), chemokines (viz. Monocyte chemoattractant protein-1 [MCP-1] and eotaxin), and leukocytes (including, NEUT, EOS, and MC), which need further examinations.

\section{Acupuncture for Inflammatory Visceral Pain}

Acupuncture is widely used in treating visceral pain, in which enhancing anti-inflammatory effect is assumed as one of the important mechanisms. Considering a large number of acupuncture forms for visceral pain, the use of this alternative therapy for inflammatory visceral pain is delineated here.

3.1. Clinical Evidence. According to a randomized controlled trial (RCT), comparing EA and medical treatment in 54 patients with chronic prostatitis/chronic pelvic pain syndrome (CP/CPPS) of category IIIB, EA $(4 \mathrm{~mA}, 99 \mathrm{~Hz})$ had significantly increased the scores of pain reduction, quality of life, and total Chronic Prostatitis Symptom Index (CPSI) compared with medical treatment [83]. These results were consistent with the reports that 47 patients with CPPS had been treated with EA (continuous wave, $3 \mathrm{~Hz}$ ). After treatment, the levels of IL-8, IL-10, and TNF $\alpha$ in prostatic fluid had decreased, and the CPSI score had dropped. Acupuncture could thus have a significant effect on the treatment of CPPS, which could achieve anti-inflammatory and analgesic effects by reducing the levels of inflammatory factors [84]. Similarly, another RCT on 144 patients had demonstrated that EA (alternating wave, $2 / 15 \mathrm{~Hz}$ ) had alleviated pain symptoms and improved quality of life concerning chronic pelvic pain in patients with the sequelae of pelvic inflammatory disease [85]. Acupuncture can further modulate the immune function in cases with irritable bowel syndrome (IBS), which is majorly manifested by downregulating the level of serum inflammatory factor IL-18, IL23 , and TNF $\alpha$ and reducing the number of MCs in the colon, to improve pain, intestinal gas, bloating, and stool consistency composite score [86]. In a pilot study, TEAS at Zusanli (ST36) and Neiguan (PC6) acupoints had similarly reduced rectal sensitivity in patients with IBS as manifested by increasing the threshold of rectal sensation of gas, desire to defecate, and pain [87]. One other pilot study had further suggested that acupuncture could effectively decrease subjective pain in pediatric patients with acute appendicitis and downregulate the white blood cell count. Therefore, this alternative medicine could be exploited as an effective nonpharmacological intervention for the treatment of acute appendicitis pain in children [88].

3.2. Animal Studies. Acupuncture has been shown to have several beneficial effects in animals with intestinal disorders. A recent study had accordingly revealed that EA $(10 \mathrm{~Hz}$, $1 \mathrm{~mA}$, plus width $0.4 \mathrm{~ms}$ ) at ST36 could reduce the production of inflammatory cytokines by activating $\alpha 7 \mathrm{nAChR}$ mediated JAK2/STAT3 signaling pathway in macrophages, thereby suppressing gastrointestinal inflammation and promoting its motility [17], which demonstrated the antiinflammatory and analgesic effects of EA through vagus nerve from the point of view of the complete nerve circuit. Another recent study showed that EA at ST36 with low intensity $(0.5 \mathrm{~mA})$ can activate sensory neurons expressing PROKR2+, thus driving the vagal-adrenal axis to play a systemic anti-inflammatory effect, and pointed out that the anti-inflammatory effect of acupuncture was related to the intensity of stimulation and the depth of acupuncture [89]. EA at ST36 also has an ameliorating effect within inflammatory environments by decreasing inducible nitric oxide synthase (iNOS) expression, increasing serum IL-10 level by square wave pulses with $100 \mathrm{~Hz}, 1 \mathrm{~mA}$, [90], and downregulating serum $\mathrm{TNF} \alpha$ and IL1- $\beta$ and colonic TNF $\alpha$ messenger ribonucleic acid (mRNA) expression by the intermittent pulse with $2 \mathrm{~Hz}$ frequency and $4 \mathrm{~mA}$ intensity [91]. EA at Tianshu (ST25), Zhongwan (CV12), and Shangjuxu (ST37) (alternating wave, 6/30 Hz) can further augment serum IL- 4 content and moderate colonic NF- $\kappa \mathrm{B}$ p65 protein expression [92], and EA $(15 / 25 \mathrm{~Hz}, 0.1-0.2 \mathrm{~mA}$, $2-4 \mathrm{~V}$ ) at ST36, Guanyuan (CV4) could modulate the balance between the splenic regulatory $\mathrm{T}$ cells and $\mathrm{T}$-helper 17 lymphocytes in ulcerative colitis [93]. Manual acupuncture at neurogenic spots with slight modification could alleviate the body weight changes and diarrhea scores and normalize the increased level of myeloperoxidase activity, $\mathrm{TNF} \alpha$, and IL- $1 \beta$ in the colitis rats [94]. 
Briefly, both clinical evidence and animal studies have proved the efficacy and reliability of acupuncture in the treatment of inflammatory visceral pain, such as CCPS, IBS, ulcerative colitis, and acute appendicitis. The effects of this form of alternative medicine on the regulation of various inflammatory factors are thus involved in its pain-relief mechanism.

\section{Anti-Inflammatory Mechanisms of Acupuncture-Related Therapies for PD}

As discussed earlier, acupuncture can bring a good effect on inflammatory visceral pain. PD, as a common visceral pain condition, is also closely related to inflammatory factors. Previous studies have mostly focused on the effects of acupuncture on PGs and analgesics. In contrast, there has been little research on the treatment of PD from an antiinflammatory perspective, to the best of the authors' knowledge. Therefore, it is necessary to explore how acupuncture affects inflammatory factors in the treatment of PD and reflect on the influence of inflammatory factors on this condition. The study findings accordingly revealed that acupuncture-related therapies have shown satisfactory effectiveness in treating dysmenorrhea by enhancing the antiinflammatory effects. The possible inflammatory mechanisms of menstrual pain and acupuncture-related therapies can thus alleviate menstrual cramps by mediating relevant inflammatory pathways, as illustrated in Figure 1.

4.1. Acupuncture. As mentioned, cytokines, chemokines, and inflammatory cells play a critical role in dysmenorrhea pathophysiology. Acupuncture has been further shown to exert a satisfactory effect on menstrual cramps; however, the mechanism of this form of alternative medicine for PD has not been fully explained. Some experimental studies have further reported that acupuncture can reduce the levels of inflammatory factors and immune cells in the rat model of dysmenorrhea.

In this respect, Zhao et al. have found that EA (dense wave, $50 \mathrm{~Hz}$ ) at CV4 and Sanyinjiao (SP6) acupoints had significantly reduced the writhing response and the contents of IL-2, 5-HT, and substance P in the serum of rats with dysmenorrhea [95]. These results were consistent with the reports by Luo et al., in which EA (dense wave, $50 \mathrm{~Hz}$ ) at CV4 and SP6 had mitigated the levels of TNF $\alpha$ and IL-1 in the serum of rats with PD, relieved uterine contractions, and alleviated pain [96], denoting that EA could alleviate menstrual pain and the mechanism was related to the reduction of peripheral inflammatory factors.

In rats with dysmenorrhea, the CD3 and CD4 levels and the ratio had further decreased, and the immune organs, namely, the thymus gland and the spleen, had shown obvious pathological changes. Li et al. have also reported that acupuncture at Zhibian (BL54) acupoint could significantly minimize the writhing response and consequently increase the levels of T lymphocyte subsets of CD3, CD4, CD4, and CD8 in the peripheral blood [97]. Similarly, Ju et al. have established that EA (dense wave, $50 \mathrm{~Hz}$ ) could not only improve the CD3 and CD4 levels but also develop pathological changes in the thymus gland and the spleen [98]. These studies have indicated that EA could relieve pain by enhancing the immune function in rats with $\mathrm{PD}$.

4.2. Moxibustion. Herbal cake-partitioned moxibustion (HM) is characterized by the combination of moxibustion with traditional Chinese medicine (TCM) and is being applied more widely than conventional moxibustion in clinical applications, especially in China [99].

$\mathrm{HM}$ can significantly reduce the writhing times, upregulate plasma beta-endorphin $\left(\beta\right.$-EP) as well as uterus $\mathrm{PGE}_{2}$ content and splenic NK cell activity, and even downregulate uterus $\mathrm{PGF}_{2 \alpha}$ levels in rats with dysmenorrhea [100]. MCs also have a wide range of relationships and functions in the neuroendocrine-immune network. HM can thus upregulate the number and degranulation rate of MCs in the Shenque (CV8) acupoint [101] and downregulate the expression of MCs in the uterus [102]. Another research had further shown that HM could achieve therapeutic effects by downregulating the high expression of upstream transient receptor potential vanilloid (TRPV) in the uterine MCs of rats with dysmenorrhea. Moreover, after pretreatment with MC membrane stabilizer (that is, sodium cromoglycate), the analgesic effect of HM could be weakened by the inhibitory effect of sodium cromoglycate on the function of MCs in the CV8 acupoint, suggesting the specific role of MCs in the analgesic effect of HM [103]. In addition, moxibustion could downregulate $\mathrm{NF}-\kappa \mathrm{B}$ expression and inhibit the release of $\mathrm{TNF} \alpha$ and IL-2 in rats with dysmenorrhea [104].

4.3. ACE Treatment. ACE is a form of TCM external therapy with some absorbable catgut suture implants into the acupoints. In addition, it is characterized by the advantages of easier operation and durable stimulation, compared with acupuncture [105]. ACE can also have a significant effect on dysmenorrhea, which may be related to its impact on inflammatory factors. Recently, studies have shown that ACE can significantly improve symptoms and pathological damage in rats with PD, downregulate NLR family pyrin domain containing 3 (NLRP3), Caspase-1, IL$1 \beta$, and IL-18 protein expression in uterine tissues [106]; upregulate plasma $\beta$-EP, uterus $\mathrm{PGE}_{2}$ content, and splenic NK cell activity; and even decrease $\mathrm{PGF}_{2 \alpha}$ content $[100,107]$ and downregulate COX-2 and NF- $\kappa \mathrm{B}$ p 65 protein expression in uterine tissues [108].

As a whole, acupuncture and its related therapies have partly shown that anti-inflammatory effects contribute to analgesic mechanisms on PD. It is worthwhile to study further to enrich the multitarget and multilink mechanism of clinical treatment of this debilitating condition.

\section{Recommendations for Future Researches}

With the understanding of the relationship between inflammation and dysmenorrhea deepens, the treatment of this common gynecological disorder from an anti-inflammatory perspective becomes promising. Given this, it is 


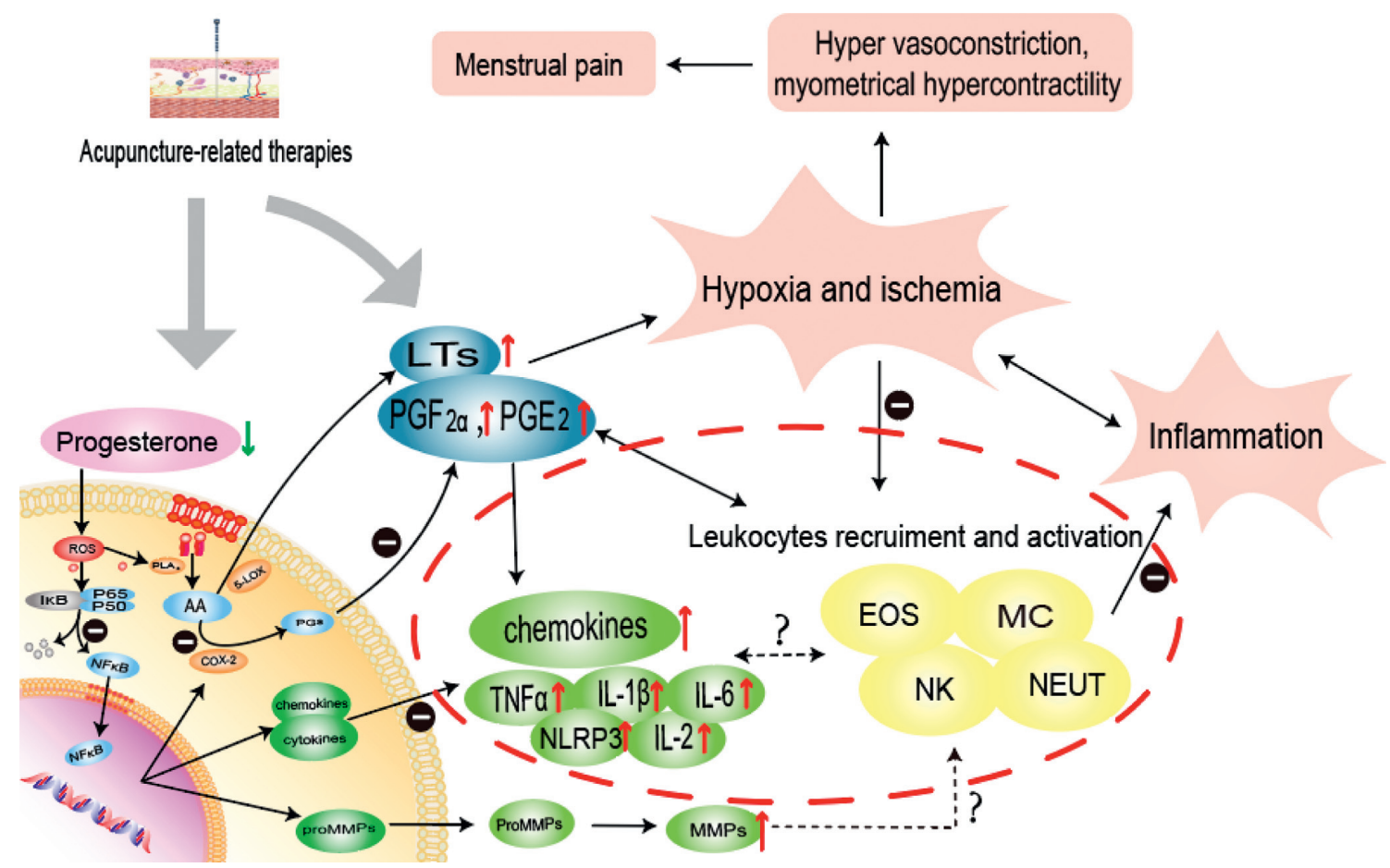

Figure 1: Possible anti-inflammatory effects of acupuncture-related therapies contributing to analgesia in menstrual pain. Progesterone withdrawal leads to the increased production of prostaglandins (PGs), leukotrienes (LTs), cytokines, chemokines, and matrix metalloproteinases (MMPs). These inflammatory factors promote the recruitment and activation of leukocytes, further increasing the inflammation and uterine hypoxia and ischemia, ultimately aggravating menstrual pain, which are considered as a vicious cycle. Acupuncture could reduce the release of PGs and downstream inflammatory cytokines by regulating nuclear factor- $\kappa \mathrm{B}(\mathrm{NF}-\kappa \mathrm{B})$ signaling pathway, further relieving the inflammatory environment of uterus and uterine ischemia and hypoxia to alleviate menstrual pain. We suggest that further complexity is added to acupuncture-induced analgesia via interactions between chemokines and leukocytes. Symbols “ $\uparrow$ " represent rising trend during menstrual pain. Symbols “-” represent inhibition of acupuncture. Abbreviations are listed at the end of the article.

speculated that enhancing anti-inflammatory effects is one of the important mechanisms of acupuncture as an alternative medicine for PD.

Based on the recent acupuncture experimental studies summarized above, we make some recommendations for future researches as follows:

1. Targeting anti-inflammatory effect of acupuncture in the treatment of PD from the perspective of chemokine-mediated inflammatory response will be a new direction to understand the mechanism of acupuncture for PD. At present, the anti-inflammatory effect of acupuncture on PD is mostly discussed from the aspects of inflammatory cells and cytokines (e.g., TNF $\alpha$, IL-1, NF- $\kappa \mathrm{B}, \mathrm{NK}$ cell activity, and $\mathrm{MC}$ ). For further studies, it is interesting to explore the effects of acupuncture on chemokines since the latest evidence has shown that the serum levels of eotaxin have an increasing trend in patients with PD [68].

(2) Optimizing acupuncture intervention methods according to classic acupuncture theory is crucial in future mechanism studies of PD. Clinical and experimental studies have indicated that alterations in needling techniques may influence the therapeutic outcomes of acupuncture [109-111]. Dysmenorrhea with the congealing-cold syndrome is the most common pattern in TCM [112]. Previous clinical and animal studies have accordingly provided evidence that transverse needling on SP6 has outperformed in relieving menstrual pain with the congealing-cold syndrome via different pathways [113-115], supporting a classic acupuncture theory of "transverse needling benefits for reinforcing yang organs of the human body". Therefore, it is recommended to conduct future acupuncture studies in line with the notion of emphasizing stimulation modes rather than acupoint selection alone. Meanwhile, it is also suggested to probe the anti-inflammatory mechanism of acupuncture with different needling methods for PD. Such studies are of great significance to increase the awareness of the benefits of acupuncture to optimize its clinical modalities for this disorder.

(3) The effects and mechanism of more convenient selfhealing approaches for PD should be investigated. Although there are numerous acupuncture-like methods to reduce menstrual cramps, a more convenient procedure for patients is urgently needed because PD interferes with the activities of daily living and work of young women. TEAS/TENS are also among the noninvasive treatment options to 
stimulate acupoints or sites on the surface of the body, which have the advantages of being safe, easy to use, and digitally operated. Studies have further shown that TENS/TEAS can treat many types of pain [116], including inflammatory pain [117] with satisfactory results [118]. A clinical study had also revealed that TEAS could decrease the levels of CXCL8, IL-1, IL-6, TNF $\alpha$, and CCL2 to relieve inflammatory responses and decrease the injuries caused by lower limb ischemia-reperfusion [119]. Another RCT had further established that TEAS could facilitate postoperative rehabilitation and even reduce stress response, and surgical inflammation in elderly patients undergoing knee surgery [120]. An animal study had correspondingly focused on the effect of TENS on the process of wound healing from the perspective of proinflammatory cytokine expression and had found that TENS had significantly mitigated the immunoreaction of TNF $\alpha$, IL- $1 \beta$, and IL-6 in the dermis compared with other treatments, indicating that TENS had shortened the healing process by inhibiting the inflammatory responses [121]. Meanwhile, studies had verified the effectiveness of TENS in reducing menstrual pain and improving the quality of life in patients with dysmenorrhea [122-124]. Compared with treatment methods such as acupuncture, TEAS has also eased restrictions on in-hospital treatment. Patients can even receive treatment at home and in the workplace. Certainly, the anti-inflammatory mechanism of TEAS/TENS with proper stimulation parameters in treating PD is worthy of further study.

\section{Conclusion}

Acupuncture and its related therapies achieve satisfied analgesic effects on $\mathrm{PD}$ via the downregulation of a wide variety of inflammatory cells and cytokines (e.g., TNF $\alpha$, IL-1, IL-2, IL-18, COX-2, NF- $\kappa$ B, NK cell activity, and MCs). The antiinflammatory effects of acupuncture may further contribute to its analgesia in the treatment of PD, so there is a need to carry out more researches to confirm it. For further studies, it is interesting to explore the effects of acupuncture on chemokine-mediated inflammation with optimized acupuncture intervention methods according to classic acupuncture theory. Additionally, the effects and mechanism of more convenient self-healing approaches such as TENS/ TEAS for PD should be investigated.

\section{Abbreviations}

PD: $\quad$ Primary dysmenorrhea

NSAIDs: Nonsteroidal anti-inflammatory drugs

EA: $\quad$ Electroacupuncture

ACE: Acupoint catgut embedding

TEAS: Electrical acupoint stimulation

TENS: Transcutaneous electrical nerve stimulation

PLA2: Phospholipase A2

AA: Arachidonic acid
PGs: Prostaglandins

LTs: Leukotrienes

COX: Cyclooxygenase

LOX: Lipoxygenase

IL: $\quad$ Interleukin

TNF $\alpha$ : Tumor necrosis factors $\alpha$

NF- $\kappa$ B: $\quad$ Nuclear factor $-\kappa B$

MMPs: Matrix metalloproteinases

EOS: $\quad$ Eosinophil

NK: $\quad$ Natural killer cells

MC: $\quad$ Mast cells

NEUT: Neutrophils

PMS: $\quad$ Premenstrual syndrome

ECP: Eosinophil cationic protein

MBP: $\quad$ Major basic protein

ROS: $\quad$ Reactive oxygen species

RCT: $\quad$ Randomized controlled trial

$\mathrm{CP} / \quad$ Chronic prostatitis/chronic pelvic pain

CPPS: syndrome

CPSI: $\quad$ Chronic Prostatitis Symptom Index

IBS: Irritable bowel syndrome

HM: Herbal cake-partitioned moxibustion

TCM: Traditional Chinese medicine

iNOS: Inducible nitric oxide synthase

mRNA: Messenger ribonucleic acid

$\beta$-EP: $\quad$ Beta-endorphin

TRPV: Transient receptor potential vanilloid

NLRP3: NLR family pyrin domain containing 3.

\section{Conflicts of Interest}

The authors declare no conflicts of interest regarding the publication of this article.

\section{Acknowledgments}

This study was supported by the National Key Research and Development Program of China (no. 2020YFC2007303), the National Natural Science Foundation of China (no. 81774417), and the Vertical Research Project of Beijing University of Chinese Medicine (no. 2018-ZXFZJJ-010).

\section{References}

[1] E.-M. Jun, S. Chang, D.-H. Kang, and S. Kim, "Effects of acupressure on dysmenorrhea and skin temperature changes in college students: a non-randomized controlled trial," International Journal of Nursing Studies, vol. 44, no. 6, pp. 973-981, 2007.

[2] M. Burnett and M. Lemyre, "No. 345-Primary dysmenorrhea consensus guideline," Journal of Obstetrics and Gynaecology Canada, vol. 39, no. 7, pp. 585-595, 2017.

[3] F. Rencz, M. Péntek, P. F. M. Stalmeier et al., "Bleeding out the quality-adjusted life years: evaluating the burden of primary dysmenorrhea using time trade-off and willingnessto-pay methods," Pain, vol. 158, no. 11, pp. 2259-2267, 2017.

[4] S. Iacovides, I. Avidon, and F. C. Baker, "What we know about primary dysmenorrhea today: a critical review," $\mathrm{Hu}$ man Reproduction Update, vol. 21, no. 6, pp. 762-778, 2015. 
[5] V. De Sanctis, A. Soliman, S. Bernasconi et al., "Primary dysmenorrhea in adolescents: prevalence, impact and recent knowledge," Pediatric Endocrinology Reviews, vol. 13, no. 2, pp. 512-520, 2015.

[6] A. Unsal, U. Ayranci, M. Tozun, G. Arslan, and E. Calik, "Prevalence of dysmenorrhea and its effect on quality of life among a group of female university students," Upsala Journal of Medical Sciences, vol. 115, no. 2, pp. 138-145, 2010.

[7] S. Y. Yu, Z. T. Lv, Q. Zhang et al., "Electroacupuncture is beneficial for primary dysmenorrhea: the evidence from meta-analysis of randomized controlled trials," Evidencebased Complementary and Alternative Medicine: eCAM, vol. 2017, Article ID 1791258, 2017.

[8] A. J. Vickers, E. A. Vertosick, G. Lewith et al., "Acupuncture for chronic pain: update of an individual patient data metaanalysis," The Journal of Pain, vol. 19, no. 5, pp. 455-474, 2018.

[9] R. B. Kelly and J. Willis, "Acupuncture for pain," American Family Physician, vol. 100, no. 2, pp. 89-96, 2019.

[10] J.-R. He, S.-G. Yu, Y. Tang, and P. Illes, "Purinergic signaling as a basis of acupuncture-induced analgesia," Purinergic Signalling, vol. 16, no. 3, pp. 297-304, 2020.

[11] Y.-P. Yu, L.-X. Ma, Y.-X. Ma et al., "Immediate effect of acupuncture at Sanyinjiao (SP6) and Xuanzhong (GB39) on uterine arterial blood flow in primary dysmenorrhea," Journal of Alternative \& Complementary Medicine, vol. 16, no. 10, pp. 1073-1078, 2010.

[12] Y.-X. Ma, X.-N. Ye, C.-Z. Liu et al., "A clinical trial of acupuncture about time-varying treatment and points selection in primary dysmenorrhea," Journal of Ethnopharmacology, vol. 148, no. 2, pp. 498-504, 2013.

[13] C. A. Smith, X. Zhu, L. He, and J Song, "Acupuncture for primary dysmenorrhoea," Cochrane Database of Systematic Reviews, no. 1, Article ID CD007854, 2011.

[14] T. Liu, J. N. Yu, B. Y. Cao, Y. Y Peng, Y. P Chen, and L Zhang, "Acupuncture for primary dysmenorrhea: a meta-analysis of randomized controlled trials," Alternative Therapies in Health and Medicine, vol. 23, no. 7, pp. 46-53, 2017.

[15] H. L. Woo, H. R. Ji, Y. K. Pak et al., "The efficacy and safety of acupuncture in women with primary dysmenorrhea," Medicine (Baltimore), vol. 97, no. 23, Article ID e11007, 2018.

[16] L. Chen, A. Xu, N. Yin et al., "Enhancement of immune cytokines and splenic CD4+ T cells by electroacupuncture at ST36 acupoint of SD rats," PLoS One, vol. 12, no. 4, Article ID e0175568, 2017.

[17] N.-N. Yang, J.-W. Yang, Y. Ye et al., "Electroacupuncture ameliorates intestinal inflammation by activating $\alpha 7 \mathrm{nAChR}$ mediated JAK2/STAT3 signaling pathway in postoperative ileus," Theranostics, vol. 11, no. 9, pp. 4078-4089, 2021.

[18] F. Lai, Y. Ren, C. Lai et al., "Acupuncture at Zusanli (ST36) for experimental sepsis: a systematic review," Evidence-based Complementary and Alternative Medicine: eCAM, vol. 2020, Article ID 3620741, 2020.

[19] K.-W. Lu, C.-K. Hsu, C.-L. Hsieh, J. Yang, and Y.-W. Lin, "Probing the effects and mechanisms of electroacupuncture at ipsilateral or contralateral ST36-ST37 acupoints on CFAinduced inflammatory pain," Scientific Reports, vol. 6, no. 1, Article ID 22123, 2016.

[20] B. X. Jin, L. L. Jin, and G.-Y. Jin, "The anti-inflammatory effect of acupuncture and its significance in analgesia," World Journal of Acupuncture-Moxibustion, vol. 29, no. 1, pp. 1-6, 2019.

[21] Y. Li, M. Yang, F. Wu et al., "Mechanism of electroacupuncture on inflammatory pain: neural-immune- endocrine interactions," Journal of traditional Chinese medicine, vol. 39, no. 5, pp. 740-749, 2019.

[22] I. S. Lee, S. Cheon, and J. Y. Park, "Central and peripheral mechanism of acupuncture analgesia on visceral pain: a systematic review," Evidence-based Complementary and Alternative Medicine: eCAM, vol. 2019, Article ID 1304152, 2019.

[23] S. Chen, S. Wang, P. Rong et al., "Acupuncture for visceral pain: neural substrates and potential mechanisms," Evidencebased Complementary and Alternative Medicine: eCAM, vol. 2014, Article ID 609594, 2014.

[24] C. A. Finn, "Implantation, menstruation and inflammation," Biological Reviews, vol. 61, no. 4, pp. 313-328, 1986.

[25] A. Tahamtan, M. Teymoori-Rad, B. Nakstad, and V. Salimi, "Anti-inflammatory MicroRNAs and their potential for inflammatory diseases treatment," Frontiers in Immunology, vol. 9, Article ID 1377, 2018.

[26] R. W. Kelly, A. E. King, and H. O. Critchley, "Inflammatory mediators and endometrial function--focus on the perivascular cell," Journal of Reproductive Immunology, vol. 57, no. 1-2, pp. 81-93, 2002.

[27] I. E. Messinis, C. I. Messini, and K. Dafopoulos, "Novel aspects of the endocrinology of the menstrual cycle," Reproductive BioMedicine Online, vol. 28, no. 6, pp. 714-722, 2014.

[28] J. A. Maybin and H. O. D. Critchley, "Progesterone: a pivotal hormone at menstruation," Annals of the New York Academy of Sciences, vol. 1221, no. 1, pp. 88-97, 2011.

[29] A. S. Coco, "Primary dysmenorrhea," American Family Physician, vol. 60, no. 2, pp. 489-496, 1999.

[30] M. Y. Dawood, "Primary dysmenorrhea," Obstetrics \& Gynecology, vol. 108, no. 2, pp. 428-441, 2006.

[31] J. Evans and L. A. Salamonsen, "Decidualized human endometrial stromal cells are sensors of hormone withdrawal in the menstrual inflammatory cascade," Biology of Reproduction, vol. 90, no. 1, p. 14, 2014.

[32] R. S. Ilad, S. D. Fleming, C. R. Murphy, and A. T. Fazleabas, "Immunohistochemical study of the ubiquitin - nuclear factor-KB pathway in the endometrium of the baboon (Papio anubis) with and without endometriosis," Reproduction, Fertility and Development, vol. 22, no. 7, pp. 1118-1130, 2010.

[33] S. Defrère, R. González-Ramos, J. C. Lousse et al., "Insights into iron and nuclear factor-kappa B (NF-kappaB) involvement in chronic inflammatory processes in peritoneal endometriosis," Histology \& Histopathology, vol. 26, no. 8, pp. 1083-1092, 2011.

[34] C. A. Finn, "Menstruation: a nonadaptive consequence of uterine evolution," The Quarterly Review of Biology, vol. 73, no. 2, pp. 163-173, 1998.

[35] M. Arenas-Hernandez, R. Romero, Y. Xu et al., "Effector and activated $\mathrm{T}$ cells induce preterm labor and birth that is prevented by treatment with progesterone," The Journal of Immunology, vol. 202, no. 9, pp. 2585-2608, 2019.

[36] J. P. Lydon, F. J. DeMayo, C. R. Funk et al., "Mice lacking progesterone receptor exhibit pleiotropic reproductive abnormalities," Genes \& Development, vol. 9, no. 18, pp. 2266-2278, 1995.

[37] B. van der Burg and P. T. V. der Saag, "Endocrinology and paracrinology," Molecular Human Reproduction, vol. 2, no. 6, pp. 433-438, 1996.

[38] H. Tan, L. Yi, N. S. Rote, W. W. Hurd, and S. Mesiano, "Progesterone receptor-A and -B have opposite effects on proinflammatory gene expression in human myometrial cells: implications for progesterone actions in human 
pregnancy and parturition," Journal of Clinical Endocrinology \& Metabolism, vol. 97, no. 5, pp. E719-E730, 2012.

[39] J. Zhang, A. L. Hampton, G. Nie, and L. A. Salamonsen, "Progesterone inhibits activation of latent matrix metalloproteinase (MMP)-2 by membrane-type 1 MMP: enzymes coordinately expressed in human Endometrium1," Biology of Reproduction, vol. 62, no. 1, pp. 85-94, 2000.

[40] B. Mofid, Z. Soltani, M. Khaksari et al., "What are the progesterone-induced changes of the outcome and the serum markers of injury, oxidant activity and inflammation in diffuse axonal injury patients?" International Immunopharmacology, vol. 32, pp. 103-110, 2016.

[41] J. A. ELdering, M. G. Nay, L. M. Hoberg, C. Longcope, and J. A. McCRACKEN, "Hormonal regulation of prostaglandin production by rhesus monkey endometrium," Journal of Clinical Endocrinology \& Metabolism, vol. 71, no. 3, pp. 596-604, 1990.

[42] X. Fan, S. Krieg, C. J. Kuo et al., "VEGF blockade inhibits angiogenesis and reepithelialization of endometrium," The FASEB Journal, vol. 22, no. 10, pp. 3571-3580, 2008.

[43] H. N. Jabbour and K. J. Sales, "Prostaglandin receptor signalling and function in human endometrial pathology," Trends in Endocrinology and Metabolism, vol. 15, no. 8, pp. 398-404, 2004.

[44] J. Evans and L. A. Salamonsen, "Inflammation, leukocytes and menstruation," Reviews in Endocrine \& Metabolic Disorders, vol. 13, no. 4, pp. 277-288, 2012.

[45] Z. Harel, "Cyclooxygenase-2 specific inhibitors in the treatment of dysmenorrhea," Journal of Pediatric and Adolescent Gynecology, vol. 17, no. 2, pp. 75-79, 2004.

[46] X. Chen, L. Weina, Y. Xingji, L. Kelycia, P. Krystyn, and F. Xin, "PGF2 $\alpha$ modulates the output of chemokines and pro-inflammatory cytokines in myometrial cells from term pregnant women through divergent signaling pathways," Molecular Human Reproduction, vol. 21, no. 7, 2015.

[47] A. E. Wallace, K. J. Sales, R. D. Catalano et al., "Prostaglandin F $2 \alpha$-F-prostanoid receptor signaling promotes neutrophil chemotaxis via chemokine (C-X-C motif) ligand 1 in endometrial adenocarcinoma," Cancer Research, vol. 69, no. 14, pp. 5726-5733, 2009.

[48] J. A. Maybin, N. Hirani, H. N. Jabbour, and H. O. D. Critchley, "Novel roles for hypoxia and prostaglandin E2 in the regulation of IL-8 during endometrial repair," American Journal Of Pathology, vol. 178, no. 3, pp. 1245-1256, 2011.

[49] I. G. Colditz, "Effect of exogenous prostaglandin E2 and actinomycin D on plasma leakage induced by neutrophilactivating peptide-1/interleukin-8," Immunology \& Cell Biology, vol. 68, no. Pt 6, pp. 397-403, 1990.

[50] P. Kannan, K.-K. Cheung, and B. W.-M. Lau, "Does aerobic exercise induced-analgesia occur through hormone and inflammatory cytokine-mediated mechanisms in primary dysmenorrhea?" Medical Hypotheses, vol. 123, pp. 50-54, 2019.

[51] J. I. Abu and J. C. Konje, "Leukotrienes in gynaecology: the hypothetical value of anti-leukotriene therapy in dysmenorrhoea and endometriosis," Human Reproduction Update, vol. 6, no. 2, pp. 200-205, 2000.

[52] E. K. S. Lee, M. R. Gillrie, L. Li et al., "Leukotriene B4mediated neutrophil recruitment causes pulmonary capillaritis during lethal fungal sepsis," Cell Host \& Microbe, vol. 23, no. 1, pp. 121-133, 2018, e4.

[53] S. Nigam, C. Benedetto, M. Zonca, I Leo-Rossberg, H Lübbert, and J Hammerstein, "Increased concentrations of eicosanoids and platelet-activating factor in menstrual blood from women with primary dysmenorrhea," Eicosanoids, vol. 4, no. 3, pp. 137-141, 1991.

[54] Z. Harel, C. Lilly, S. Riggs, R. Vaz, and J. Drazen, "Urinary leukotriene (LT) E4 in adolescents with dysmenorrhea: a pilot study," Journal of Adolescent Health, vol. 27, no. 3, pp. 151-154, 2000.

[55] Z. Harel, S. Riggs, R. Vaz, P. Flanagan, and D. Harel, "The use of the leukotriene receptor antagonist montelukast (Singulair) in the management of dysmenorrhea in adolescents," Journal of Pediatric and Adolescent Gynecology, vol. 17, no. 3, pp. 183-186, 2004.

[56] B. Grulović, M. R. Pucelj, M. Krnić, and V Kokić, "Impact of prostaglandin F2-alpha and tumor necrosis factor-alpha (TNF-alpha) on pain in patients undergoing thermal balloon endometrial ablation," Collegium Antropologicum, vol. 37, no. 4, pp. 1185-1190, 2013.

[57] H. Ma, M. Hong, J. Duan et al., "Altered cytokine gene expression in peripheral blood monocytes across the menstrual cycle in primary dysmenorrhea: a case-control study," PLoS One, vol. 8, no. 2, Article ID e55200, 2013.

[58] M. Marchini, B. Manfredi, L. Tozzi, P. Sacerdote, A. Panerai, and L. Fedele, "Immunology: mitogen-induced lymphocyte proliferation and peripheral blood mononuclear cell $\beta$-endorphin concentrations in primary dysmenorrhoea," $\mathrm{Hu}$ man Reproduction, vol. 10, no. 4, pp. 815-817, 1995.

[59] D. H. Que, W. H. Chen, F. P. Jiang, P. Fei, and Y. Ke, "Mechanism of Danggui Sini Decoction in treatment of primary dysmenorrhea based on network pharmacology and molecular docking [in Chinese]," Zhongguo Zhongyao Zazhi, vol. 46, no. 4, pp. 855-864, 2021.

[60] H. Y. Dogru, A. Z. Ozsoy, N. Karakus, I. B. Delibas, C. K. Isguder, and S. Yigit, "Association of genetic polymorphisms in TNF and MIF gene with the risk of primary dysmenorrhea," Biochemical Genetics, vol. 54, no. 4, pp. 457-466, 2016.

[61] B. W. Whitcomb, S. L. Mumford, N. J. Perkins et al., "Urinary cytokine and chemokine profiles across the menstrual cycle in healthy reproductive-aged women," Fertility and Sterility, vol. 101, no. 5, pp. 1383-1391, 2014.

[62] E. R. Bertone-Johnson, A. G. Ronnenberg, S. C. Houghton et al., "Association of inflammation markers with menstrual symptom severity and premenstrual syndrome in young women," Human Reproduction, vol. 29, no. 9, pp. 1987-1994, 2014.

[63] L. Konecna, M. S. Yan, L. E. Miller, J. Schölmerich, W. Falk, and R. H. Straub, "Modulation of IL-6 production during the menstrual cycle in vivo and in vitro," Brain, Behavior, and Immunity, vol. 14, no. 1, pp. 49-61, 2000.

[64] P. Henriet, H. P. Gaide Chevronnay, and E. Marbaix, "The endocrine and paracrine control of menstruation," Molecular and Cellular Endocrinology, vol. 358, no. 2, pp. 197-207, 2012.

[65] M.-L. Yeh, H.-H. Chen, E. C. So, and C.-F. Liu, "A study of serum malondialdehyde and interleukin-6 levels in young women with dysmenorrhea in Taiwan," Life Sciences, vol. 75, no. 6, pp. 669-673, 2004.

[66] J. R. White, C. Imburgia, E. Dul et al., "Cloning and functional characterization of a novel human CC chemokine that binds to the CCR3 receptor and activates human eosinophils," Journal of Leukocyte Biology, vol. 62, no. 5, pp. $667-675,1997$.

[67] U. Forssmann, C. Stoetzer, M. Stephan et al., "Inhibition of CD26/dipeptidyl peptidase IV enhances CCL11/eotaxin- 
mediated recruitment of eosinophils in vivo," The Journal of Immunology, vol. 181, no. 2, pp. 1120-1127, 2008.

[68] E. Gul and E. Celik Kavak, "Eotaxin levels in patients with primary dysmenorrhea," Journal of Pain Research, vol. 11, pp. 611-613, 2018.

[69] L. A. Salamonsen and L. J. Lathbury, "Endometrial leukocytes and menstruation," Human Reproduction Update, vol. 6 , no. 1, pp. 16-27, 2000.

[70] Z. Harel, "Dysmenorrhea in adolescents and young adults: an update on pharmacological treatments and management strategies," Expert Opinion on Pharmacotherapy, vol. 13, no. 15, pp. 2157-2170, 2012.

[71] A. Bahrami, H. Bahrami-Taghanaki, Z. Khorasanchi et al., "Menstrual problems in adolescence: relationship to serum vitamins $\mathrm{A}$ and $\mathrm{E}$, and systemic inflammation," Archives of Gynecology and Obstetrics, vol. 301, no. 1, pp. 189-197, 2020.

[72] Ö. Kaplan, M. Nazıroğlu, M. Güney, and M. Aykur, "Nonsteroidal anti-inflammatory drug modulates oxidative stress and calcium ion levels in the neutrophils of patients with primary dysmenorrhea," Journal of Reproductive Immunology, vol. 100, no. 2, pp. 87-92, 2013.

[73] M. Jeziorska, L. A. Salamonsen, and D. E. Woolley, "Mast cell and eosinophil distribution and activation in human endometrium throughout the menstrual Cycle1," Biology of Reproduction, vol. 53, no. 2, pp. 312-320, 1995.

[74] C. Poropatich, M. Rojas, and S. G. Silverberg, "Polymorphonuclear leukocytes in the endometrium during the normal menstrual cycle," International Journal of Gynecological Pathology, vol. 6, no. 3, pp. 230-234, 1987.

[75] J. M. D. Araújo, L. A. S. Silva, F. B. Felix, E. A Camargo, and R Grespan, "CCR3 antagonist impairs estradiol-induced eosinophil migration to the uterus in ovariectomized mice," Brazilian Journal of Medical and Biological Research, vol. 53, no. 1, Article ID e8659, 2020.

[76] M. C. O’Donnell, S. J. Ackerman, G. J. Gleich, and L. L. Thomas, "Activation of basophil and mast cell histamine release by eosinophil granule major basic protein," Journal of Experimental Medicine, vol. 157, no. 6, pp. 1981-1991, 1983.

[77] M. E. Akbalik, N. Liman, H. Sagsoz, and B. Guney Saruhan, "Tissue distribution of some immune cells in bovine reproductive tract during follicular and luteal phase," $M i$ croscopy Research and Technique, vol. 81, no. 3, pp. 315-331, 2018.

[78] E. Bytautiene, Y. P. Vedernikov, G. R. Saade, R. Romero, and R. E. Garfield, "Degranulation of uterine mast cell modifies contractility of isolated myometrium from pregnant women," American Journal of Obstetrics and Gynecology, vol. 191, no. 5, pp. 1705-1710, 2004.

[79] R. Garfield, E. Bytautiene, Y. Vedernikov, J. Marshall, and R. Romero, "Modulation of rat uterine contractility by mast cells and their mediators," American Journal of Obstetrics and Gynecology, vol. 183, no. 1, pp. 118-125, 2000.

[80] M. J. Hamilton, S. M. Frei, and R. L. Stevens, "The multifaceted mast cell in inflammatory bowel disease," Inflammatory Bowel Diseases, vol. 20, no. 12, pp. 2364-2378, 2014.

[81] A. J. Hueber, D. L. Asquith, A. M. Miller et al., "Cutting edge: mast cells express IL-17a in rheumatoid arthritis synovium," The Journal of Immunology, vol. 184, no. 7, pp. 3336-3340, 2010.

[82] T. Lipitsä, A. Naukkarinen, and I. T. Harvima, "Mast cell tryptase and chymase in the progress of cutaneous vasculitis," Archives of Dermatological Research, vol. 307, no. 10, pp. 917-924, 2015.
[83] E. V. Küçük, F. Y. Suçeken, A. Bindayı, U Boylu, F. F Onol, and E Gümüş, "Effectiveness of acupuncture on chronic prostatitis-chronic pelvic pain syndrome category IIIB patients: a prospective, randomized, nonblinded, clinical trial," Urology, vol. 85, no. 3, pp. 636-640, 2015.

[84] S. Y. Yuan, Z. Qin, D. S. Liu, Y. Wei-Qiang, Z. Zhao-Lei, and L. Shen-Guo, "Acupuncture for chronic pelvic pain syndromes (CPPS) and its effect on cytokines in prostatic fluid," Zhongguo Zhen Jiu, vol. 31, no. 1, pp. 11-14, 2011, in Chinese.

[85] Z. Liang, X. Wang, Y. H. Liu, Z. Dong-Mei, and S. Ling, "Analgesic effect of electroacupuncture on chronic pelvic pain in patients with sequelae of pelvic inflammatory disease," Zhongguo Zhen Jiu, vol. 41, no. 4, pp. 395-399, 2021, in Chinese.

[86] X.-P. Ma, J. Hong, C. P. An, Z. Dan, H. Yan, and W. HuanGan, "Acupuncture-moxibustion in treating irritable bowel syndrome: how does it work?" World Journal of Gastroenterology, vol. 20, no. 20, pp. 6044-6054, 2014.

[87] J. Xing, B. Larive, N. Mekhail, and E Soffer, "Transcutaneous electrical acustimulation can reduce visceral perception in patients with the irritable bowel syndrome: a pilot study," Alternative Therapies in Health and Medicine, vol. 10, no. 1, pp. 38-42, 2004.

[88] A. L. Nager, M. Kobylecka, P. K. Pham, L. Johnson, and J. I. Gold, "Effects of acupuncture on pain and inflammation in pediatric emergency department patients with acute appendicitis: a pilot study," Journal of Alternative \& Complementary Medicine, vol. 21, no. 5, pp. 269-272, 2015.

[89] S. Liu, Z. Wang, Y. Su et al., "A neuroanatomical basis for electroacupuncture to drive the vagal-adrenal axis," Nature, vol. 598, no. 7882, pp. 641-645, 2021.

[90] A. C. A. d. M. Goes, F. M. M. Pinto, G. C. Fernandes et al., "Electroacupuncture ameliorates experimental colitis induced by TNBS through activation of interleukin-10 and inhibition of iNOS in mice," Acta Cirurgica Brasileira, vol. 29, no. 12, pp. 787-793, 2014.

[91] L. Tian, Y. X. Huang, M. Tian, G. Wei, and C. Qing, "Downregulation of electroacupuncture at ST36 on TNF- $\alpha$ in rats with ulcerative colitis," World Journal of Gastroenterology, vol. 9, no. 5, pp. 1028-1033, 2003.

[92] L. X. Ji, Y. T. Cheng, L. P. Yan, W. Hai-Jun, and J. Xiao-Fei, "Effects of electroacupuncture at acupoints "changbingfang" on serum IL-4 content and colonic NF-kappaB p65 expression in rats with ulcerative colitis," Zhen Ci Yan Jiu, vol. 38, no. 1, pp. 26-30, 2013, in Chinese.

[93] C. Y. Wang, L. L. Zeng, Y. Geng, W. Xiang, and Z. He-jiao, "Effect of electroacupuncture stimulation of "guanyuan" (CV 4) and "Zusanli" (ST 36) on spleen lymphocytes treg/Th 17 immune balance in ulcerative colitis mice," Zhen Ci Yan Jiu, vol. 41, no. 1, pp. 55-59, 2016, in Chinese.

[94] D.-H. Kim, Y. Ryu, D. H. Hahm et al., "Acupuncture points can be identified as cutaneous neurogenic inflammatory spots," Scientific Reports, vol. 7, no. 1, Article ID 15214, 2017.

[95] Z. F. Zhao and C. Z. Tang, "Effect of electroacupuncture at Guanyuan (CV4) and Sanyinjiao (SP6) on serum IL-2,5-HT and substance P in dysmenorrhea model rats," Global Trad Chin Med, vol. 10, no. 5, pp. 541-543, 2017, in Chinese.

[96] Y. W. Luo, Q. J. Zhang, Y. X. Zheng, and C. Qing, "Effect of electroacupuncture on serum levels of TNF- $\alpha$ IL-1 in experimental dysmenorrhea rats," Chin J of Basic Med in Trad Chin Med, no. 9, pp. 712-716, 2008, in Chinese.

[97] L. Y. Li, P. Yan, and X. F. JIN, "Effect of acupuncture at zhibian point on $\mathrm{T}$ lymphocyte subsets of primary 
dysmenorrhea model rats," J of Clin Acu and Mox, vol. 32, no. 3, pp. 70-72, 2016, in Chinese.

[98] Y. L. Ju, L. Wang, and J. Z. Liu, "Influences of electroacupuncture, moxibustion and catgut embedding on T lymphocyte subgroups in dysmenorrhea rats," Shanghai $J$ of Acu and Mox, no. 9, pp. 43-45, 2007, in Chinese.

[99] S.-Y. Wang, J.-M. Zhao, C.-L. Zhou et al., "Herbal cakepartitioned moxibustion inhibits colonic autophagy in Crohn's disease via signaling involving distinct classes of phosphatidylinositol 3-kinases," World Journal of Gastroenterology, vol. 26, no. 39, pp. 5997-6014, 2020.

[100] B. P. Chen, S. S. Qi, and J. Cui, "Herbal-cake-patitioned moxibustion of "Shenque" (CV8) has a relative specific effect in relieving abdominal pain and in regulating neuroendocrine-immune network in primary dysmenorrhea rats," Zhen Ci Yan Jiu, vol. 44, no. 2, pp. 120-124+135, 2019, in Chinese.

[101] S. J. Li, X. N. Zhang, and F. J. Ma, "Effect of umbilical therapy on Shenque acupoint mast cells of treating cold coagulation and blood stasis type of dysmenorrhea model rats," Lishizhen Med Mater Med Res, vol. 28, no. 9, pp. 2288-2290, 2017, in Chinese.

[102] S. J. Li, Y. Sun, X. N. Zhang, M. Sun, and S. Song, "Effect of umbilical therapy on uterine mast cells of treating cold coagulation and blood stasis type of dysmenorrhea model rats," Liaoning J of Trad Chin Med, vol. 45, no. 8, pp. 1751-1754+1789, 2018, in Chinese.

[103] S. J. Li, Y. Sun, and Y. H. Pan, "Effects of mast cell function on umbilical therapy for uterine transient receptor potential vanillin pathway in rats with dysmenorrhea of cold congelation and blood stasis sydrome," Chin J of Trad Chin Med Phar, vol. 33, no. 10, pp. 4646-4649, 2018, in Chinese.

[104] B. Y. Zhou, X. L. Wu, and H. B. Wu, "Effect of mediated thread moxibustion of Zhuang medicine on NF- $\kappa \mathrm{B}$ and related factors in rats with dysmenorrhea of cold-damp stagnation pattern," Guangxi Journal of Traditional Chinese Medicine, vol. 41, no. 5, pp. 59-61, 2018, in Chinese.

[105] J. Wu, Q. Fu, S. Yang, H. Wang, and Y Li, "Efficacy and safety of acupoint catgut embedding for diarrhea-predominant irritable bowel syndrome and constipation-predominant irritable bowel syndrome: a systematic review and metaanalysis," Evidence-based Complementary and Alternative Medicine: eCAM, vol. 2020, Article ID 5812320, 2020.

[106] Y. Q. Wang, L. Y. Chen, B. Q. Mo, W. Xiao Xian, and L. Yu, "Acupoint catgut embedding improves dysmenorrhea by suppressing activation of NLRP3 inflammasomes in uterine tissues in primary dysmenorrhea rats," Zhen Ci Yan Jiu, vol. 44, no. 7, pp. 481-485, 2019, in Chinese.

[107] B. P. Chen, J. Chen, J. Cui, and Y. Xiao Fang, "Effects of the acupoint catgut embedding on nerve-endocrine-immune network in dysmenorrhea rats," Zhen Ci Yan Jiu, vol. 43, no. 1, pp. 29-33, 2018, in Chinese.

[108] W. J. Tang, Y. Q. Wang, and B. Tang, "Effect of acupoint catgut embedding on levels of PG-related factors and NF- $\kappa \mathrm{B}$ proteins in uterine tissues of rats with primary dysmenorrhea," Zhen Ci Yan Jiu, vol. 45, no. 7, pp. 548-551+556, 2020, in Chinese.

[109] E. Kim, J. H. Cho, W. S. Jung, S Lee, and S. C Pak, "Effect of acupuncture on heart rate variability in primary dysmenorrheic women," The American Journal of Chinese Medicine, vol. 39, no. 2, pp. 243-249, 2011.

[110] J.-X. Wang, L.-X. Ma, J.-D. Mu et al., "Anti-spastic effect induced by waggle needling correlates with KCC2-GABAA pathway in post-stroke spasticity rats," Neuroscience Letters, vol. 750, Article ID 135810, 2021.
[111] J.-X. Wang, J.-D. Mu, L.-X. Ma et al., "Waggle needling wields preferable neuroprotective and anti-spastic effects on post-stroke spasticity rats by attenuating $\gamma$-aminobutyric acid transaminase and enhancing $\gamma$-aminobutyric acid," NeuroReport, vol. 31, no. 10, pp. 708-716, 2020.

[112] Y.-q. Liu, L.-x. Ma, J.-m. Xing et al., "Does traditional Chinese medicine pattern affect acupoint specific Effect? Analysis of data from a multicenter, randomized, controlled trial for primary dysmenorrhea," Journal of Alternative \& Complementary Medicine, vol. 19, no. 1, pp. 43-49, 2013.

[113] A. Mohammadi, L.-X. Ma, Y. Yang, Y. Song, and J.-X. Wang, "Immediate analgesic effect of perpendicular needling or transverse needling at SP 6 in primary dysmenorrhea: a pilot study," Medical Acupuncture, vol. 31, no. 4, pp. 207-217, 2019.

[114] Y. Y. Gan, L. X. Ma, and W. Y. Yu, "Effects of transverse and perpendicular needling at Sanyinjiao (SP6) on the uterine microcirculation and NO, NOS in cold congealing dysmenorrhea rats," Chin J of Trad Chin Med Phar, vol. 36, no. 3, pp. 1663-1667, 2021, in Chinese.

[115] A. Mohammadi, M. R. Afshari Fard, L. Ma et al., "Analgesic effects and hemodynamic mechanisms of perpendicular and transverse needling at Sanyinjiao (SP 6) in patients with primary dysmenorrhea: a randomized controlled trial," Journal of Traditional Chinese Medical Sciences, vol. 8, no. 3, pp. 248-256, 2021.

[116] K. L. Sato, L. S. Sanada, M. D. d. Silva, R. Okubo, and K. A. Sluka, "Transcutaneous electrical nerve stimulation, acupuncture, and spinal cord stimulation on neuropathic, inflammatory and, non-inflammatory pain in rat models," The Korean Journal of Pain, vol. 33, no. 2, pp. 121-130, 2020.

[117] T. C. do Carmo Almeida, F. W. Dos Santos Figueiredo, V. C. Barbosa Filho, A. Luiz Carlos de, and A. F. Fernando Luiz, "Effects of transcutaneous electrical nerve stimulation on proinflammatory cytokines: systematic review and metaanalysis," Mediators of Inflammation, vol. 2018, Article ID 1094352, 2018.

[118] J. M. DeSantana, D. M. Walsh, C. Vance, B. A. Rakel, and K. A. Sluka, "Effectiveness of transcutaneous electrical nerve stimulation for treatment of hyperalgesia and pain," Current Rheumatology Reports, vol. 10, no. 6, pp. 492-499, 2008.

[119] Y. Mo, S. Chen, L. Yang et al., "The effect of transcutaneous electrical acupoint stimulation on inflammatory response in patients undergoing limb ischemia-reperfusion," Mediators of Inflammation, vol. 2017, Article ID 8369737, 2017.

[120] Y. L. Chi, W. L. Zhang, F. Yang, F Su, and Y. K Zhou, "Transcutaneous electrical acupoint stimulation for improving postoperative recovery, reducing stress and inflammatory responses in elderly patient undergoing knee surgery," The American Journal of Chinese Medicine, vol. 47, no. 7, pp. 1445-1458, 2019.

[121] S. G. Gürgen, O. Sayın, F. Cetin, and A Tuç Yücel, "Transcutaneous electrical nerve stimulation (TENS) accelerates cutaneous wound healing and inhibits pro-inflammatory cytokines," Inflammation, vol. 37, no. 3, pp. 775-784, 2014.

[122] M. Elboim-Gabyzon and L. Kalichman, "Transcutaneous electrical nerve stimulation (TENS) for primary dysmenorrhea: an overview," International Journal of Women's Health, vol. 12, pp. 1-10, 2020.

[123] M. I. Arik, H. Kiloatar, B. Aslan, and M. Icelli, "The effect of tens for pain relief in women with primary dysmenorrhea: a systematic review and meta-analysis," Explore, vol. 2541, 2020 . 
[124] G. R. Lauretti, R. Oliveira, F. Parada, and A. L. Mattos, "The new portable transcutaneous electrical nerve stimulation device was efficacious in the control of primary dysmenorrhea cramp pain," Neuromodulation: Technology at the Neural Interface, vol. 18, no. 6, pp. 522-527, 2015. 